\title{
Designing metal hemispheres on silicon ultrathin film solar cells for plasmonic light trapping
}

\author{
Tongchuan Gao, ${ }^{1}$ Erica Stevens, ${ }^{2}$ Jung-kun Lee, ${ }^{2}$ and Paul W. Leu ${ }^{1, *}$ \\ ${ }^{1}$ Department of Industrial Engineering, University of Pittsburgh, Pittsburgh, Pennsylvania 15261, USA \\ ${ }^{2}$ Department of Mechanical Engineering \& Materials Science, University of Pittsburgh, Pittsburgh, Pennsylvania 15261, USA \\ *Corresponding author: pleu@pitt.edu
}

Received May 20, 2014; revised June 17, 2014; accepted June 18, 2014; posted June 20, 2014 (Doc. ID 212368); published August 4, 2014

\begin{abstract}
We systematically investigate the design of two-dimensional silver (Ag) hemisphere arrays on crystalline silicon (c-Si) ultrathin film solar cells for plasmonic light trapping. The absorption in ultrathin films is governed by the excitation of Fabry-Perot TEM ${ }_{m}$ modes. We demonstrate that metal hemispheres can enhance absorption in the films by (1) coupling light to c-Si film waveguide modes and (2) exciting localized surface plasmon resonances (LSPRs). We show that hemisphere arrays allow light to couple to fundamental $\mathrm{TE}_{m}$ and $\mathrm{TM}_{m}$ waveguide modes in c-Si film as well as higher-order versions of these modes. The near-field light concentration of LSPRs also may increase absorption in the c-Si film, though these resonances are associated with significant parasitic absorption in the metal. We illustrate how Ag plasmonic hemispheres may be utilized for light trapping with $22 \%$ enhancement in short-circuit current density compared with that of a bare $100 \mathrm{~nm}$ thick c-Si ultrathin film solar cell. () 2014 Optical Society of America

OCIS codes: (040.5350) Photovoltaic; (310.6628) Subwavelength structures, nanostructures; (250.5403) Plasmonics; (160.2100) Electro-optical materials.

http://dx.doi.org/10.1364/OL.39.004647
\end{abstract}

One particular light trapping strategy for crystalline silicon (c-Si) thin film solar cells involves the utilization of metallic nanostructures that support plasmonic effects, such as localized surface plasmon resonances (LSPRs) and surface plasmon polaritons. Various metal nanogrids [1-3] and nanoparticles [ 4-7] have been demonstrated to enhance absorption in a variety of solar cell structures. To take full advantage of plasmonic light trapping, sophisticated tuning is essential because of the trade-off between the enhancement introduced by the excitation of plasmonic effects and the additional reflection and parasitic absorption losses from the metal.

In this Letter, we investigate metal hemisphere arrays on c-Si ultrathin film solar cells for plasmonic light trapping. While randomly distributed or individual nanoparticles have been studied previously, there have yet to be any studies on regular arrays of nanoparticles. We focus on hemispheres, which have been shown to exhibit higher near-field coupling than spherical nanoparticles [4]. We find that plasmonic hemispheres may be utilized to enhance the short-circuit current density of c-Si ultrathin film solar cells of $100 \mathrm{~nm}$ thickness by over $20 \%$. The absorption in bare thin films is governed by Fabry-Perot (FP) modes. We show that plasmonic hemispheres on the front surface of these films introduce additional absorption resonances due to (1) coupling of light to waveguide modes in c-Si thin films and (2) excitation of LSPRs. We also evaluate angle-dependent optical properties of plasmonic hemispheres to offer further physical insight into various resonance modes and light trapping properties of these hemispheres.

Figure 1 is a schematic of the system we studied. The photoactive region consists of a c-Si ultrathin film with thickness $t_{\mathrm{Si}}$. A two-dimensional (2D) array of silver (Ag) hemispheres sits on top of the silicon thin film. The arrays are defined by the diameter $d$ of the hemisphere and pitch $a$ of the square lattice. We investigated c-Si thin films with $t_{\mathrm{Si}}=100 \mathrm{~nm}$, hemispheres with diameters $d$ from 50 to $500 \mathrm{~nm}$, and pitches $a$ from 50 to $500 \mathrm{~nm}$, where $d \leq a$. The two parameters were varied in $5 \mathrm{~nm}$ increments.

We performed electrodynamic simulations by solving Maxwell's equations using the finite-difference timedomain method []. We used a perfectly matched layer for the top boundary condition of the simulation supercell and a perfectly reflecting boundary, representing an ideal metal back contact, for the bottom boundary condition. The side boundary conditions of the supercell were set to be antisymmetric and symmetric in the direction of the electric and magnetic fields, respectively, to model the periodic nature of the $\mathrm{Ag}$ hemispheres. We also utilized a nonuniform simulation mesh with a finer mesh near interfaces and a larger mesh in bulk regions. The optical constants for the c-Si and Ag were taken from experimental results [9]. The simulation data was recorded at 150 linearly spaced points with respect to the frequency between $\lambda=300$ and $1200 \mathrm{~nm}$.

The position-dependent absorption per unit volume $A(\mathbf{r}, \lambda)$ was calculated from the divergence of the Poynting vector $\mathbf{P}$ normalized over the incoming radiation power $P_{\text {in }}(\lambda)$ :

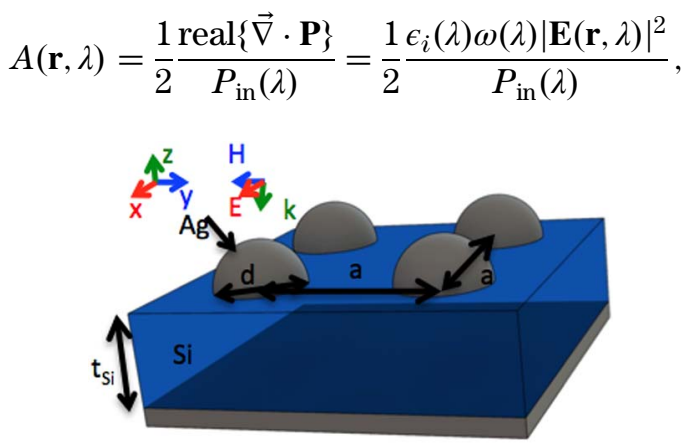

Fig. 1. Schematic of the plasmonic solar cell structure. A square array of hemispheres of diameter $d$ and pitch $a$ sits on top of a c-Si thin film of thickness $t_{\mathrm{Si}}$ on a perfect backreflector. 
where $\lambda$ is the free-space wavelength, $\epsilon_{i}(\lambda)$ is the imaginary part of the permittivity, $\omega(\lambda)=2 \pi c / \lambda$ is the photon angular frequency, $c$ is the speed of light, and $\mathbf{E}(\mathbf{r}, \lambda)$ is the electric field vector as the function of position and wavelength. The absorption spectra $A(\lambda)$ of the Si photoactive region was obtained by integrating position-dependent absorption per unit volume over $\mathrm{Si}$ volume, $A(\lambda)=$ $\int A(\mathbf{r}, \lambda) \mathrm{d} V_{\mathrm{Si}}$. This eliminates any parasitic absorption that may occur in the Ag hemispheres and that does not contribute to solar efficiency. Assuming that each absorbed photon generates one electron-hole pair and that all photogenerated carriers are collected, the shortcircuit current density is

$$
J_{\mathrm{sc}}=q \int_{0}^{\lambda_{g}} \frac{I(\lambda)}{\lambda} A(\lambda) \mathrm{d} \lambda,
$$

where $\lambda_{g}=1107 \mathrm{~nm}$ is the wavelength corresponding to the bandgap of $\mathrm{c}-\mathrm{Si}\left(E_{g}=1.12 \mathrm{eV}\right)$, and $I(\lambda)$ is the solar irradiance under the global $37^{\circ}$ tilt air mass 1.5 spectrum.

Figure 2(a) plots the absorption spectra of a $t_{\mathrm{Si}}=$ $100 \mathrm{~nm}$ c-Si film on a perfect backreflector. For thin film systems, FP modes are supported where the resonant wavelengths are sensitive to thickness. Maxwell's equations may be solved with appropriate boundary conditions to determine that FP modes occur for an Si thin film on a perfect conductor when the following condition is satisfied:

$$
\tan \left(n_{\mathrm{Si}} k t_{\mathrm{Si}}\right)=-n_{\mathrm{Si}} i,
$$

where $k$ is the free-space wavenumber, and $n_{\mathrm{Si}}$ is the refractive index of c-Si. The FP modes [10] are degenerate $\mathrm{TE}$ and TM modes, and thus labeled as $\mathrm{TEM}_{m}$ modes with mode number $m$. The thin film absorption resonances for $m=2$ and 1 at $\lambda=410$ and $550 \mathrm{~nm}$, respectively, are marked in Fig. 2(a) with white dashed lines. The electric

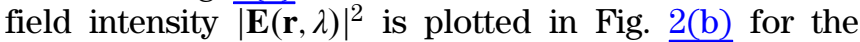
(i) $\mathrm{TEM}_{2}$ and (ii) $\mathrm{TEM}_{1}$ modes. These resonant modes are characterized by $m / 2+1 / 4$ wavelengths in the transverse direction of the planar film, where the electric field intensity is maximum at the front surface and 0 at the

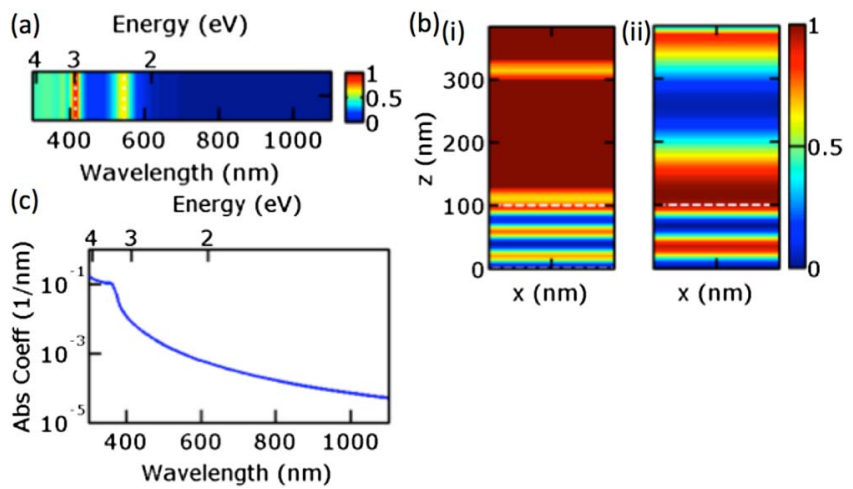

Fig. 2. (a) Absorption spectra of $t_{\mathrm{Si}}=100 \mathrm{~nm} \mathrm{c-Si} \mathrm{film} \mathrm{on} \mathrm{a}$ perfect backreflector. The FP modes are marked with white dashed lines and correspond to the $\mathrm{TEM}_{2}$ and $\mathrm{TEM}_{1}$ modes at $\lambda=410$ and $550 \mathrm{~nm}$, respectively. (b) Electric field intensity $|\mathbf{E}(\mathbf{r}, \lambda)|^{2}$ of the (i) $\mathrm{TEM}_{2}$ and (ii) $\mathrm{TEM}_{1}$ modes. The c-Si layer is the region $z=0$ to $100 \mathrm{~nm}$. (c) Absorption coefficient of bulk c-Si from [9]. back metal interface. Light absorption is increased by constructive interference in the c-Si thin film at these particular wavelengths. In contrast, bulk c-Si has a relatively featureless absorption spectrum [Fig. 2(c)].

Two-dimensional arrays of $\mathrm{Ag}$ hemispheres with $d$ from 50 to $500 \mathrm{~nm}$ and $a$ from 50 to $500 \mathrm{~nm}$ were subsequently investigated for plasmonic light trapping. The highest enhancement in $J_{\mathrm{sc}}$ was determined to be exhibited when $d=170 \mathrm{~nm}$ and $a=245 \mathrm{~nm}$. Figure 3 illustrates absorption in the $t_{\mathrm{Si}}=100 \mathrm{~nm}$ c-Si film on a perfect backreflector with $d=170 \mathrm{~nm}$ Ag hemispheres on the front surface. Figure 3(a) plots the absorption in the c-Si as a function of the pitch $a$ from 170 to $500 \mathrm{~nm}$ and the free-space wavelength. Several additional absorption peaks are seen due to the presence of the $\mathrm{Ag}$ hemispheres on the top surface. Apart from the FP modes of the film, which are again plotted with white dashed lines, introducing Ag hemisphere arrays on the front of c-Si thin film allows for excitation of waveguide modes and plasmons. The TE waveguide modes for a thin film of $\mathrm{Si}$ on a perfect conductor satisfy

$$
k_{\mathrm{Si}} \cot \left(k_{\mathrm{Si}} t_{\mathrm{Si}}\right)=i k_{x},
$$

where $k_{\mathrm{Si}}$ and $k_{x}$ are the transverse wavenumbers in c-Si and the air, respectively, $k_{\mathrm{Si}}=\left(n_{\mathrm{Si}}^{2} k^{2}-\beta^{2}\right)^{1 / 2}$ and $k_{x}=\left(k^{2}-\beta^{2}\right)^{1 / 2}$. $\beta$ is the propagation constant. TM modes for a thin film of c-Si on a perfect conductor satisfy

$$
i k_{\mathrm{Si}} \tan \left(k_{\mathrm{Si}} t_{\mathrm{Si}}\right)=n_{\mathrm{Si}}^{2} k_{x} .
$$

The periodicity of Ag hemisphere arrays allows light to couple to waveguide modes in c-Si thin film when $\beta=2 \pi / a$. Equations (4) and (5) reduce to Eq. (3) when $\beta=0$. The analytical solutions of the $\mathrm{TM}_{0}, \mathrm{TE}_{0}, \mathrm{TM}_{1}$,
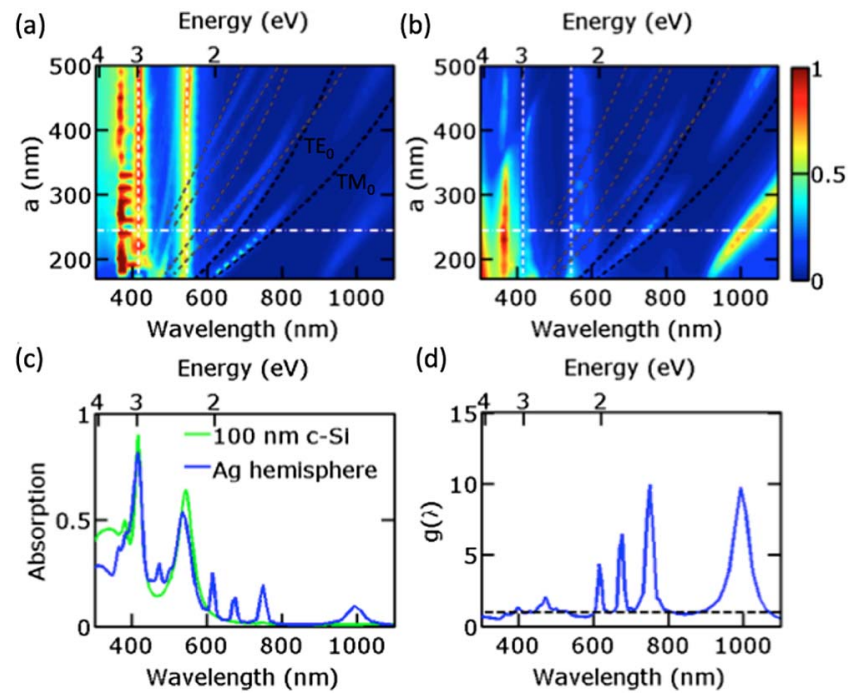

Fig. 3. Absorption in (a) c-Si and (b) Ag hemispheres for a 2D Ag hemisphere array of diameter $d=170 \mathrm{~nm}$ and different pitches $a$ from 170 to $400 \mathrm{~nm}$ on $t_{\mathrm{Si}}=100 \mathrm{~nm}$ c-Si thin film on a perfect backreflector. (c) Absorption as a function of the wavelength for $100 \mathrm{~nm}$ thick c-Si layer with and without $\mathrm{Ag}$ hemispheres at $a=245 \mathrm{~nm}$ (indicated by the white dasheddotted line in (a). (d) Absorption enhancement $g(\lambda)$ due to the hemispheres as a function of wavelength. 
and $\mathrm{TE}_{1}$ mode are plotted with black dashed lines in Fig. 3(a). As $a \rightarrow \infty$ and $\beta \rightarrow 0$, the $\mathrm{TE}_{0}$ and $\mathrm{TM}_{0}$ modes converge to the $\mathrm{TEM}_{0}$ mode at $\lambda=1350 \mathrm{~nm}$, and the $\mathrm{TE}_{1}$ and $\mathrm{TM}_{1}$ modes converge to the $\mathrm{TEM}_{1}$ mode at $\lambda=550 \mathrm{~nm}$. Higher-order modes also may be excited at lower wavelengths (higher energies) when the propagation constant matches the reciprocal lattice constant, $\beta=2 \pi / a \sqrt{p^{2}+q^{2}}$, where $p$ and $q$ are integers. The $\mathrm{TM}_{0}$ and $\mathrm{TE}_{0}$ modes are plotted for $p=1$ and $q=1$ as well as for $p=2$ and $q=0$ (or $p=0, q=2$ ) with gray-dashed lines. The simulated modes are slightly blueshifted compared with the analytical solution due to the presence of metal on the front side of the c-Si.

Figure 3(b) plots absorption in the Ag hemispheres as a function of pitch $a$ from 170 to $500 \mathrm{~nm}$ and the free-space wavelength. The FP modes and waveguide modes are plotted with dashed lines as before. Light is efficiently coupled into the FP modes where there is little absorption in the metal. Increased absorption in the metal can be seen when the incoming light is coupled into the $\mathrm{TM}_{m}$ waveguide modes. In addition, pitch-dependent absorption enhancement in the $\mathrm{Si}$ and metal hemispheres can be seen at longer wavelengths in the neighborhood of $\lambda=1000 \mathrm{~nm}$. This absorption enhancement corresponds to an LSPR, based on analysis of the electric field intensity, and its angle dependence, which will be later discussed. The absorption is enhanced in the metal and c-Si at this wavelength due to strong local field enhancement around the metal hemisphere.

The maximum short-circuit current density was obtained at $a=245 \mathrm{~nm}$, which is indicated by the white dashed-dotted line in Fig. 3(a). The absorption spectra for this pitch $A_{h}(\lambda)$ is plotted in Fig. 3(c) along with the absorption spectra for the c-Si thin film without $\mathrm{Ag}$ hemispheres $A_{\text {bare }}(\lambda)$. The short-circuit current density for $\mathrm{c}-\mathrm{Si}$ with the selected $\mathrm{Ag}$ hemisphere array is $J_{\mathrm{sc}}=6.1 \mathrm{~mA} / \mathrm{cm}^{2}$, which is a $22 \%$ enhancement compared with $J_{\mathrm{sc}}=5.0 \mathrm{~mA} / \mathrm{cm}^{2}$ for the bare c-Si thin film. The absorption enhancement $g(\lambda)=A_{h}(\lambda) / A_{\text {bare }}(\lambda)$ is plotted in Fig. 3(d). Four enhancement peaks at $\lambda=620$, 680,750 , and $1000 \mathrm{~nm}$ can be seen. The peaks reside in the long wavelength range with an enhancement of about 10 at $\lambda=750$ and $1000 \mathrm{~nm}$.

The three resonance modes at $\lambda=620,680$, and $750 \mathrm{~nm}$ correspond to the excitation of waveguide modes in the c-Si and exhibit absorption enhancements of 4.3, 6.5 , and 9.9 times, respectively, compared with that of a $t_{\mathrm{Si}}=100 \mathrm{~nm}$ c-Si thin film. Figure 4 plots the electric field intensity $|\mathbf{E}(\mathbf{r}, \lambda)|^{2}$ at $\lambda=$ (a) 620 , (b) 680, and (c) $750 \mathrm{~nm}$. The electric field of the incident light is in the positive $x$ direction, and the magnetic field is in the negative $y$ direction in these plots, as indicated in Fig. 1. The top row in Fig. 4 shows the electric field intensity through the center of the c-Si at $z=50 \mathrm{~nm}$, and the bottom row shows the electric field intensity through the center of one of the hemispheres along $y=0$. The mode at $\lambda=$ $620 \mathrm{~nm}$ corresponds to a $\mathrm{TM}_{0}$ mode with $p=1$ and $q=1$. The mode at $\lambda=750 \mathrm{~nm}$ is also a $\mathrm{TM}_{0}$ mode, but with $p=0$ and $q=1$ (or $p=1$ and $q=0$ ). The profiles of these modes in the $z$ direction resemble each other, but the mode at $\lambda=620 \mathrm{~nm}$ has periodicity in the $x$ and $y$ directions, while the mode at $\lambda=750 \mathrm{~nm}$ only has

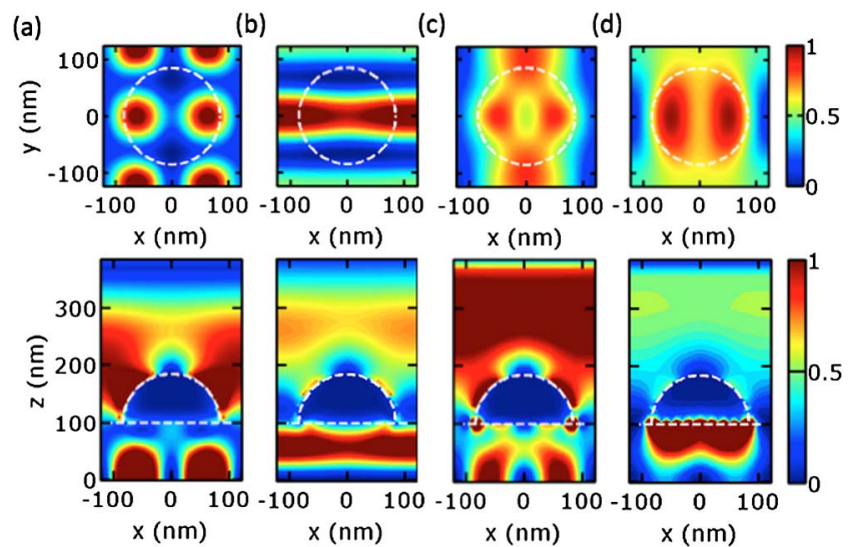

Fig. 4. Electric field intensity profile $|\mathbf{E}(\mathbf{r}, \lambda)|^{2}$ at $\lambda=$ (a) 620 , (b) 680 , (c) $750 \mathrm{~nm}$, and (d) $1000 \mathrm{~nm}$. The top row shows a slice in the $x-y$ plane at $z=50 \mathrm{~nm}$, and the bottom row shows an $x-y$ slice at $y=0$. (a) and (c) are $\mathrm{TM}_{0}$ modes, while (b) is a $\mathrm{TE}_{0}$ mode. (d) is an LSPR. All the plots have been normalized to the same color bar.

periodicity in the $x$ direction. In addition, the mode at $\lambda=$ $680 \mathrm{~nm}$ is a $\mathrm{TE}_{0}$ mode with $p=0$ and $q=1$ (or $p=1$ and $q=0)$. This mode has one period in the $y$ direction. The metal hemispheres on the front surface of the c-Si film allows light to couple to waveguide modes in the c-Si film, where the mode energy is concentrated in the $\mathrm{Si}$ layer, leading to absorption enhancement.

The electric field intensity at $\lambda=1000 \mathrm{~nm}$ is shown in Fig. 4(d), which corresponds to an LSPR. The resonance mode at $\lambda=1000$ exhibits an enhancement of about 10 times compared with that of a $t_{\mathrm{Si}}=100 \mathrm{~nm}$ c-Si thin film. This concentration of light inside the Si film is induced by a surface plasmon with a dipolar character, where the electric field intensity decays exponentially into the c-Si layer away from the Ag and c-Si interface. The parasitic absorption in the metal is significant at this wavelength and about $76 \%$. This mode shifts to higher wavelengths (lower energies) with increasing pitch due to the electromagnetic interaction between neighboring hemispheres. We found that this resonance peak exhibits some pitch dependence for pitches below $500 \mathrm{~nm}$.

Finally, we studied the angle-dependent absorption behavior in hemisphere array structures. Figure 5 plots the angle dependence of absorption from $0^{\circ}$ to $35^{\circ}$ for the structure with $d=170 \mathrm{~nm}$ and $a=245 \mathrm{~nm}$ for (a) TEand (b) TM-polarized incident light. The fundamental $\mathrm{TM}_{0}$ mode at $\lambda=750$ is only dependent on the incidence angle for TM-incident light, while the fundamental $\mathrm{TE}_{0}$ mode at $\lambda=680 \mathrm{~nm}$ is only dependent on the incidence angle for TE-incident light. In contrast, the higher-order $\mathrm{TM}_{0}$ mode at $\lambda=620 \mathrm{~nm}$ is dependent on incidence angle for both TE- and TM-incidence light, since $p=1$ and $q=1$. The LSPR is independent of the incidence angle for TE- and TM-incident light. The short-circuit current density is shown in Fig. 5 and is almost constant for oblique incident light. Furthermore, the angle dependence of the short-circuit current density is similar for TE- and TM-polarized incident light.

In summary, we investigated the design of $2 \mathrm{D} \mathrm{Ag} \mathrm{hemi-}$ sphere arrays for plasmonic c-Si ultrathin film solar cells. Structures with a broad range of parameters were 


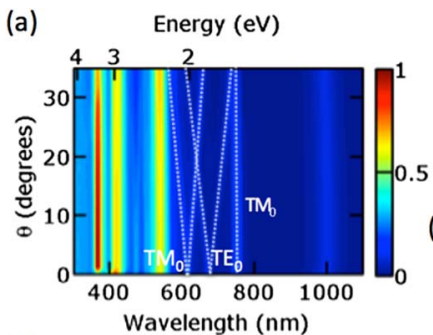

(b)

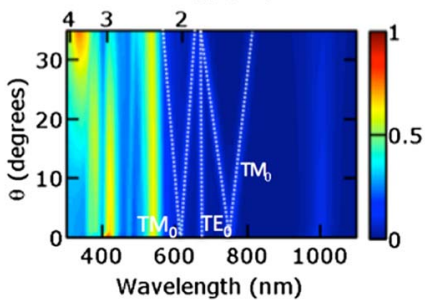

(c)

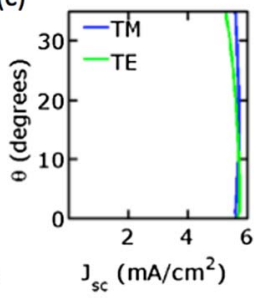

Fig. 5. Absorption spectra of $d=170 \mathrm{~nm}$ and $a=245 \mathrm{~nm} \mathrm{Ag}$ hemisphere array on $t_{\mathrm{Si}}=100 \mathrm{~nm}$ thin film on a perfect backreflector as a function of the wavelength for (a) TE-incident light and (b) TM-incident light. Waveguide modes are indicated by white dashed lines. (c) Short-circuit current density $J_{\mathrm{sc}}$ as a function of incident angle.

systematically studied, and the best structure was observed to have a $22 \%$ enhancement in short-circuit current density compared to that of a bare c-Si solar cell. Three types of resonance modes: (1) FP; (2) waveguide; and (3) LSPR are supported by the studied structure. FP modes may be excited in bare c-Si solar cells, but the introduction of 2D hemisphere arrays introduces waveguide and LSPR modes that lead to enhanced ab- sorption compared with that of bare c-Si solar cells. The resonance modes can be conveniently tuned by varying the Ag hemisphere array pitch. We also studied angle dependence of the absorption in these structures and demonstrated how absorption resonances shift with the incidence angle.

This work was supported in part by NSF grant no. 1233151. Computing resources were provided by the Center for Simulation and Modeling at the University of Pittsburgh. Erica Stevens would like to thank NSF NUE grant no. 1242075 and the Mascaro Center for Sustainable Innovation for support.

\section{References}

1. V. E. Ferry, L. A. Sweatlock, D. Pacifici, and H. A. Atwater, Nano Lett. 8, 4391 (2008).

2. R. A. Pala, J. White, E. Barnard, J. Liu, and M. L. Brongersma, Adv. Mater. 21, 3504 (2009).

3. I. Kim, T. S. Lee, D. S. Jeong, W. S. Lee, W. M. Kim, and K.-S. Lee, Opt. Express 21, A669 (2013)

4. K. R. Catchpole and A. Polman, Opt. Express 16, 21793 (2008).

5. S. Pillai, K. R. Catchpole, T. Trupke, and M. A. Green, J. Appl. Phys. 101, 093105 (2007).

6. A. Centeno, J. Breeze, B. Ahmed, H. Reehal, and N. Alford, Opt. Lett. 35, 76 (2010).

7. L. Chen, W. C. H. Choy, and W. E. I. Sha, Appl. Phys. Lett. 102, 251112 (2013).

8. S. Zivanovic, K. Yee, and K. Mei, IEEE Trans. Microwave Theor. Tech. 39, 471 (1991).

9. E. D. Palik, Handbook of Optical Constants of Solids (Academic, 1997).

10. Y. Yu and L. Cao, Opt. Express 20, 13847 (2012). 